\title{
Crossings between curves with many tangencies
}

\author{
Jacob Fox ${ }^{\dagger, \star}$, Fabrizio Frati ${ }^{\ddagger}, J_{\text {ńnos Pach }}^{\sharp, \star \star}$, and Rom Pinchasi ${ }^{\diamond}$ \\ $\dagger$ Department of Mathematics, Princeton University, Princeton, NJ \\ jacobfox@math.princeton.edu \\ ‡ Dipartimento di Informatica e Automazione, Roma Tre University, Italy \\ frati@dia.uniroma3.it \\ \# EPFL Lausanne, Switzerland and Rényi Institute Budapest, Hungary \\ pach@cims.nyu.edu \\ $\diamond$ Mathematics Department, Technion - \\ Israel Institute of Technology, Haifa 32000, Israel. \\ room@math.technion.ac.il
}

\begin{abstract}
Let $\mathcal{A}$ and $\mathcal{B}$ be two families of two-way infinite $x$-monotone curves, no three of which pass through the same point. Assume that every curve in $\mathcal{A}$ lies above every curve in $\mathcal{B}$ and that there are $m$ pairs of curves, one from $\mathcal{A}$ and the other from $\mathcal{B}$, that are tangent to each other. Then the number of proper crossings among the members of $\mathcal{A} \cup \mathcal{B}$ is at least $(1 / 2-o(1)) m \ln m$. This bound is almost tight.
\end{abstract}

\section{Introduction}

Studying the incidence structure of a family of curves in the plane is a classical theme in combinatorial geometry with many applications in computational geometry. Venn diagrams were introduced in the 19th century to analyze logical relationships between various statements $[9,7]$. The incidence structure of non-overlapping circular disks was investigated by Koebe [2], while Erdős [4] raised several questions about tangencies between possibly overlapping congruent disks, including his famous problem on unit distances: How many pairs of points can be at distance one from each other in a set of $n$ points in the plane? In other words, how many tangencies can occur among $n$ unit diameter disks in the plane? These are hard questions, see [5] for a survey.

An equally tantalizing innocent-looking question was asked by Richter and Thomassen [6]. We say that two closed curves $\gamma_{1}$ and $\gamma_{2}$ in the plane properly cross if they share at least one point $p$ (called a crossing point) such that $\gamma_{1}$ passes from one side to the other side of $\gamma_{2}$ in a small neighborhood of $p$. We say that two closed curves $\gamma_{1}$ and $\gamma_{2}$ in the plane touch or are tangent to each other, if they share exactly one point. This point is called the point of tangency of the two curves. We say that two closed curves are intersecting if they have at least

\footnotetext{
* Research supported by an NSF Graduate Research Fellowship and a Princeton Centennial Fellowship.

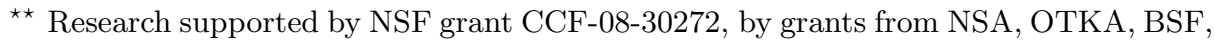
and SNF.
} 
one point in common. A family $\mathcal{F}$ of closed curves is intersecting if every pair of them is intersecting. The family $\mathcal{F}$ is in general position if any two of its members share only a finite number of points and no three members pass through the same point. According to the Richter-Thomassen conjecture, any intersecting family of $n$ closed curves in general position in the plane determines a total of at least $(1-o(1)) n^{2}$ crossing points. This, of course, holds automatically if no two curves of the family touch each other, because then the number of crossing points is at least $2\left(\begin{array}{l}n \\ 2\end{array}\right)$. Therefore, in order to settle the problem, we have to analyze families of curves with many tangencies.

In this note, we take the first step in this direction by studying the system of tangencies between two intersecting families $\mathcal{A}$ and $\mathcal{B}$ of curves in general position, with the property that no curve in $\mathcal{A}$ properly crosses any curve in $\mathcal{B}$ (see Fig. 1). In this case, we are going to prove that, if $m$ denotes the number of pairs of touching curves $(\alpha, \beta)$ with $\alpha \in \mathcal{A}$ and $\beta \in \mathcal{B}$, the total number of crossing points in $\mathcal{F}=\mathcal{A} \cup \mathcal{B}$ divided by $m$ tends to infinity, as $m \rightarrow \infty$. Consequently, if $|\mathcal{F}|=n$ and $m>\varepsilon n^{2}$ for some $\varepsilon>0$, then the total number of crossing points in $\mathcal{F}$ is superquadratic in $n$.

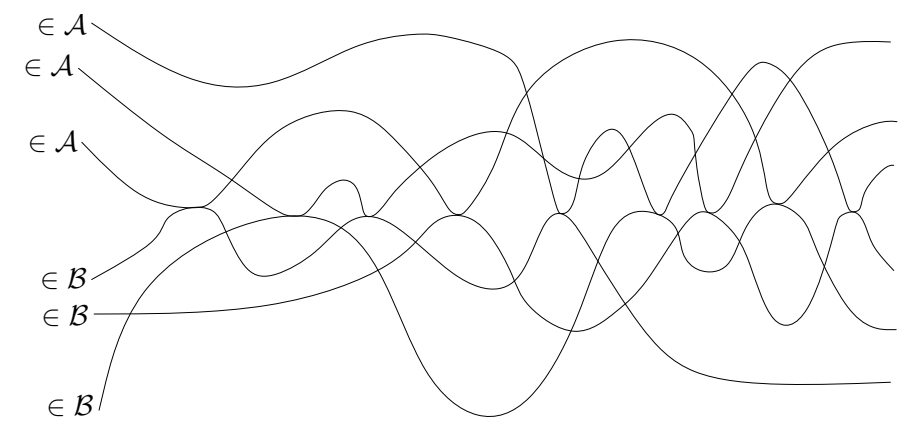

Fig. 1. Two intersecting families $\mathcal{A}$ and $\mathcal{B}$ of curves in general position such that no curve in $\mathcal{A}$ properly crosses any curve in $\mathcal{B}$.

For aesthetical reasons, we formulate our results for two-way infinite $x$ monotone curves, that is, for graphs $\gamma_{f}$ of continuous functions $f: \mathbb{R} \rightarrow \mathbb{R}$. For simplicity, in the sequel, we use the term curve in this sense. We say that a curve $\gamma_{f}$ lies above a curve $\gamma_{g}$ if $f(x) \geq g(x)$ for all $x \in \mathbb{R}$.

For any family $\mathcal{F}$ of curves in general position, let $\mathrm{CN}(\mathcal{F})$ denote the number of crossing points.

Our main result is the following.

Theorem 1. Let $\mathcal{A}$ and $\mathcal{B}$ be two families of two-way infinite $x$-monotone curves such that $\mathcal{A} \cup \mathcal{B}$ is in general position. Assume that every curve in $\mathcal{A}$ lies above every curve in $\mathcal{B}$ and that there are $m$ pairs of curves, one from $\mathcal{A}$ and the other from $\mathcal{B}$, that touch. Then the sum of the numbers of proper crossings among the 
members of $\mathcal{A}$ and among the members of $\mathcal{B}$ satisfies

$$
C N(\mathcal{A})+C N(\mathcal{B}) \geq\left(\frac{1}{2}-o(1)\right) m \ln m,
$$

where the o(1) term goes to 0 as $m$ tends to $\infty$.

We say that $\mathcal{A}$ and $\mathcal{B}$ completely touch if every member of $\mathcal{A}$ touches every member of $\mathcal{B}$.

Theorem 2. For every $n>2$, there exist two completely touching n-member families $\mathcal{A}$ and $\mathcal{B}$ of two-way infinite $x$-monotone curves such that $\mathcal{A} \cup \mathcal{B}$ is in general position, every curve in $\mathcal{A}$ lies above every curve in $\mathcal{B}$, and

$$
C N(\mathcal{A})+C N(\mathcal{B}) \leq\left(\frac{3}{4}+o(1)\right) n^{2} \log _{2} n .
$$

Comparing Theorems 1 and 2, we obtain that if $c(n)$ denotes the minimum number of crossing points in the union $\mathcal{A} \cup \mathcal{B}$ of two completely touching $n$ member families of curves, $\mathcal{A}$ and $\mathcal{B}$, such that all the members of $\mathcal{A}$ are above all the members of $\mathcal{B}$, then we have:

$$
(1-o(1)) n^{2} \ln n \leq c(n) \leq\left(\frac{3}{4}+o(1)\right) n^{2} \log _{2} n=\left(\frac{3}{4 \ln 2}+o(1)\right) n^{2} \ln n .
$$

This shows that Theorem 1 is tight up to a multiplicative factor of roughly $\frac{3}{4 \ln 2} \approx 1.082$.

In Sections 2 and 3 of this note, we establish Theorems 1 and 2, respectively. In the final section, we make some concluding remarks. In particular, we formulate a combinatorial result of independent interest on alternations in certain sequences over finite alphabets (Theorem 3), which can also be used to prove Theorem 1.

\section{Levels - Proof of Theorem 1}

The lower $k$-level of a family $\mathcal{F}$ of curves is the closure of the set of all points that lie on exactly one member of $\mathcal{F}$ and strictly above exactly $k-1$ members (see Fig. 2). Let $\ell_{k}(\mathcal{F})$ denote the number of all proper crossings among members of $\mathcal{F}$ that lie on the lower $k$-level of $\mathcal{F}$. Analogously, the upper $k$-level of a family $\mathcal{F}$ of curves is the closure of the set of all points that lie on exactly one member of $\mathcal{F}$ and strictly below exactly $k-1$ members. Let $u_{k}(\mathcal{F})$ denote the number of all proper crossings among members of $\mathcal{F}$ that lie on the upper $k$-level of $\mathcal{F}$. Note that each proper crossing among two members of a family $\mathcal{F}$ of curves in general position lies on two consecutive levels, so that we have

$$
\sum_{k=1}^{|\mathcal{F}|} \ell_{k}(\mathcal{F})=\sum_{k=1}^{|\mathcal{F}|} u_{k}(\mathcal{F})=2 \mathrm{CN}(\mathcal{F}) .
$$

Theorem 1 can be easily deduced from the following lemma. 


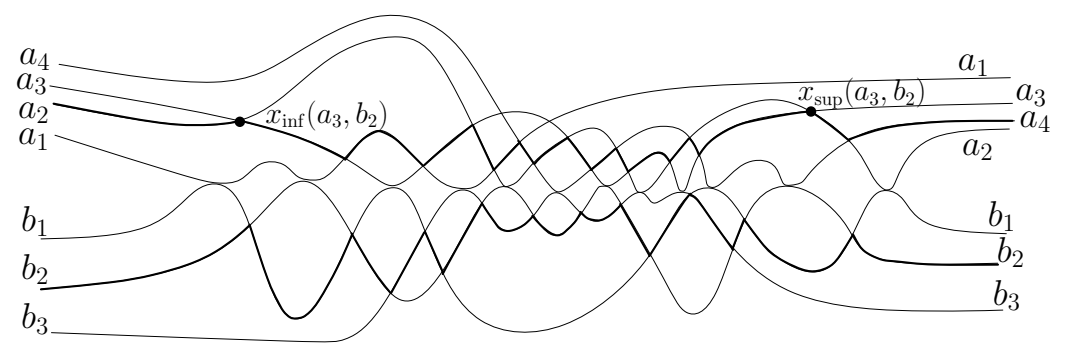

Fig. 2. Two families $\mathcal{A}$ and $\mathcal{B}$ of curves, with $|\mathcal{A}|=4$ and $|\mathcal{B}|=3$. The lower 2-level of $\mathcal{A}$ and the upper 2-level of $\mathcal{B}$ are shown by thick lines. Black dots show $x_{\text {inf }}\left(a_{3}, b_{2}\right)$ and $x_{\text {sup }}\left(a_{3}, b_{2}\right)$ when $k=2$.

Lemma 1. Let $k>1$ and $\mathcal{A}$ and $\mathcal{B}$ be two families of two-way infinite $x$ monotone curves, each of cardinality at least $k$, such that $\mathcal{A} \cup \mathcal{B}$ is in general position. Assume that every curve in $\mathcal{A}$ lies above every curve in $\mathcal{B}$ and that there are $m$ pairs of curves, one from $\mathcal{A}$ and the other from $\mathcal{B}$, that touch. Then, we have

$$
\ell_{1}(\mathcal{A})+u_{1}(\mathcal{B}) \geq m-1
$$

and

$$
\ell_{k}(\mathcal{A})+u_{k}(\mathcal{B}) \geq 2 \frac{m}{k}-4 k .
$$

Proof: We may assume without loss of generality that all crossing points between members of $\mathcal{A} \cup \mathcal{B}$ have distinct $x$-coordinates and that all of these values belong to the open interval $0<x<1$.

Note that, as $x$ varies between the $x$-coordinates of two consecutive points at which a member of $\mathcal{A}$ touches a member of $\mathcal{B}$, the lowest curve of $\mathcal{A}$ or the highest curve of $\mathcal{B}$ must change. This yields the inequality

$$
\ell_{1}(\mathcal{A})+u_{1}(\mathcal{B}) \geq m-1 .
$$

Fix $k>1$. For any $0 \leq \xi \leq 1$ which is not the $x$-coordinate of an intersection point, let $\mathcal{A}_{k}(\xi)$ denote the $k$ th lowest curve in $\mathcal{A}$ at the vertical line $x=\xi$ and let $\mathcal{B}_{k}(\xi)$ denote the $k$ th highest curve in $\mathcal{B}$ at the vertical line $x=\xi$. Analogously, $\mathcal{A}_{\leq k}(\xi)$ denotes the family consisting of the $k$ lowest curves in $\mathcal{A}$ at the vertical line $x=\xi$ and $\mathcal{B}_{\leq k}(\xi)$ denotes the family consisting of the $k$ highest curves in $\mathcal{B}$ at the vertical line $x=\xi$.

For $\xi=0$ or $\xi=1$, the number of pairs $(a, b) \in \mathcal{A}_{\leq k}(\xi) \times \mathcal{B}_{\leq k}(\xi)$ is $k^{2}$. A pair $(a, b) \in \mathcal{A} \times \mathcal{B}$ is said to be internally touching if $a$ and $b$ touch each other and

$$
(a, b) \notin\left(\mathcal{A}_{\leq k}(0) \times \mathcal{B}_{\leq k}(0)\right) \cup\left(\mathcal{A}_{\leq k}(1) \times \mathcal{B}_{\leq k}(1)\right) .
$$

Let $I$ stand for the number of internally touching pairs $(a, b)$. Clearly, we have $I \geq m-2 k^{2}$. For any internally touching pair $(a, b)$, let (see Fig. 2)

1. $x_{\text {inf }}(a, b)$ be the infimum of all $x$-values for which $\mathcal{A}_{k}(x)=a$ and $b \in \mathcal{B}_{\leq k}(x)$, or $a \in \mathcal{A}_{\leq k}(x)$ and $\mathcal{B}_{k}(x)=b$, and let 
2. $x_{\text {sup }}(a, b)$ be the supremum of all $x$-values for which $\mathcal{A}_{k}(x)=a$ and $b \in$

$\mathcal{B}_{\leq k}(x)$, or $a \in \mathcal{A}_{\leq k}(x)$ and $\mathcal{B}_{k}(x)=b$.

Obviously, we have $x_{\text {inf }}(a, b)<x_{\text {sup }}(a, b)$ as the $x$-coordinate of the touching point between $a$ and $b$ lies strictly between these two numbers. It is also clear that the numbers $x_{\text {inf }}(a, b)$ and $x_{\text {sup }}(a, b)$ are $x$-coordinates of crossing points lying on the $k$ th lowest level of $\mathcal{A}$ or on the $k$ th highest level of $\mathcal{B}$.

For any $0<\xi<1$, there are at most $k$ internally touching pairs $(a, b)$ with $x_{\text {inf }}(a, b)=\xi$. Indeed, for any $a \in \mathcal{A}$ such that $a=\mathcal{A}_{k}(\xi+\varepsilon)$, say, for all sufficiently small $\varepsilon>0$, all curves $b \in \mathcal{B}$ with $x_{\text {inf }}(a, b)=\xi$ must belong to the set $\mathcal{B}_{\leq k}(\xi)$. This is a set of size $k$. Thus, the number of distinct $x$-coordinates $\xi$ at which either $\mathcal{A}_{k}(\xi)$ or $\mathcal{B}_{k}(\xi)$ changes is at least $2 I / k$. That is, we have

$$
\ell_{k}(\mathcal{A})+u_{k}(\mathcal{B}) \geq \frac{2 I}{k} \geq 2 \frac{m-2 k^{2}}{k}=2 \frac{m}{k}-4 k .
$$

A similar argument was used in [1].

Now we are in a position to establish Theorem 1.

Proof of Theorem 1: Assume without loss of generality that $|\mathcal{A}| \geq|\mathcal{B}|$ and that every curve in $\mathcal{A} \cup \mathcal{B}$ participates in at least one touching pair. This implies that any two members of $\mathcal{A}$ properly cross at least once and any two members of $\mathcal{B}$ properly cross at least once. Hence, we have

$$
\mathrm{CN}(\mathcal{A})+\mathrm{CN}(\mathcal{B}) \geq\left(\begin{array}{c}
|\mathcal{A}| \\
2
\end{array}\right)+\left(\begin{array}{c}
|\mathcal{B}| \\
2
\end{array}\right)
$$

This completes the proof in the special case where $m \leq|\mathcal{A}|^{2} / \ln |\mathcal{A}|$, because then the term $\left(\begin{array}{c}|\mathcal{A}| \\ 2\end{array}\right)$ already exceeds the desired lower bound. In particular, since the total number $m$ of touching pairs is at most $|\mathcal{A}||\mathcal{B}|$, we are done if $|\mathcal{B}| \leq$ $|\mathcal{A}| / \ln |\mathcal{A}|$.

From now on, we can assume that

$$
m>|\mathcal{A}|^{2} / \ln |\mathcal{A}|
$$

and

$$
|\mathcal{A}| / \ln |\mathcal{A}| \leq|\mathcal{B}| \leq|\mathcal{A}|
$$

Let $\varepsilon>0$ be a very small constant. Set $K=m^{\frac{1}{2}-\varepsilon}$, and add up $\ell_{k}(\mathcal{A})+$ $u_{k}(\mathcal{B})$ for all $1 \leq k \leq K$. Note that we can apply Lemma 1 , since the last two inequalities imply that $K \leq|\mathcal{B}|$. In view of (1), we obtain

$$
\begin{aligned}
\mathrm{CN}(\mathcal{A})+\mathrm{CN}(\mathcal{B}) & \geq \frac{1}{2} \sum_{k=1}^{K}\left(\ell_{k}(\mathcal{A})+u_{k}(\mathcal{B})\right) \geq \frac{1}{2}\left(m-1+\sum_{k=2}^{K}\left(2 \frac{m}{k}-4 k\right)\right) \\
& \geq \frac{1}{2}\left(m-2 K(K+1)+3+2 m \sum_{k=2}^{K} \frac{1}{k}\right)=\left(\frac{1}{2}-\epsilon-o(1)\right) m \ln m
\end{aligned}
$$

Letting $\epsilon \rightarrow 0$, we can conclude that $\mathrm{CN}(\mathcal{A})+\mathrm{CN}(\mathcal{B})$ is at least $\left(\frac{1}{2}-o(1)\right) m \ln m$, as required. 


\section{Constructive upper bound - Proof of Theorem 2}

Let $c(n)$ denote the minimum number of crossing points in the union of any two completely touching $n$-member families of curves $\mathcal{A} \cup \mathcal{B}$, where all members of $\mathcal{A}$ are above all members of $\mathcal{B}$.

We need the following:

Lemma 2. For any pair of positive integers $i$ and $j$, we have

$$
c(i j) \leq i^{2} c(j)+j^{2} c(i) .
$$

Proof: Let $\left(\mathcal{A}^{\prime}, \mathcal{B}^{\prime}\right)$ be a pair of completely touching $i$-member families of curves with

$$
\mathrm{CN}\left(\mathcal{A}^{\prime}\right)+\mathrm{CN}\left(\mathcal{B}^{\prime}\right)=c(i) .
$$

Replace each curve $\gamma \in \mathcal{A}^{\prime} \cup \mathcal{B}^{\prime}$ by $j$ curves that closely follow $\gamma$. For any $\alpha \in \mathcal{A}^{\prime}$ and for any $\beta \in \mathcal{B}^{\prime}$, let each of the $j$ curves corresponding to $\alpha$ touch each of the $j$ curves corresponding to $\beta$ in a small neighborhood of the point where $\alpha$ and $\beta$ touch each other. This can be achieved by introducing $c(j)$ crossings near each point of tangency between $\alpha$ and $\beta$. Apart from the crossings introduced in the neighborhoods of these points, the $j$ new curves corresponding to an "old" curve $\gamma \in \mathcal{A}^{\prime} \cup \mathcal{B}^{\prime}$ are disjoint.

Denote the family of $i j$ curves obtained from the members of $\mathcal{A}^{\prime}$ by $\mathcal{A}$, and the family of $i j$ curves obtained from $\mathcal{B}^{\prime}$ by $\mathcal{B}$. Since the number of tangencies between $\mathcal{A}^{\prime}$ and $\mathcal{B}^{\prime}$ is $i^{2}$, there are at most $i^{2} c(j)$ crossings among the members of $\mathcal{A} \cup \mathcal{B}$ that occur near these touching points. On the other hand, in a small neighborhood of each crossing between two members of $\mathcal{A}^{\prime}$ or two members of $\mathcal{B}^{\prime}$, we create $j^{2}$ crossings in $\mathcal{A}$ or in $\mathcal{B}$. Therefore, there are $j^{2} c(i)$ crossings among members of $\mathcal{A} \cup \mathcal{B}$ that occur near crossings in $\mathcal{A}^{\prime}$ or $\mathcal{B}^{\prime}$. In view of the fact that each crossing in $\mathcal{A} \cup \mathcal{B}$ occurs in a small neighborhood of either a touching point or a crossing point in $\mathcal{A}^{\prime} \cup \mathcal{B}^{\prime}$, we obtain that $c(i j) \leq \mathrm{CN}(\mathcal{A})+\mathrm{CN}(\mathcal{B}) \leq$ $i^{2} c(j)+j^{2} c(i)$, as required.

Using the fact $c(2)=3$, by repeated application of Lemma 2 with $j=2$ and $i=2,2^{2}, \ldots, 2^{k-1}$. we obtain that $c\left(2^{k}\right) \leq \frac{3}{4} k 4^{k}$. Starting with a completely touching pair of 2-member families of curves, after $k-1$ iterations we obtain a completely touching pair $(\mathcal{A}, \mathcal{B})$ of $2^{k}$-member families with $m=2^{2 k}$ touching pairs. Thus, there exists a configuration with only $\frac{3}{4} k 4^{k}=\frac{3}{8} m \log _{2} m$ crossings, meeting the requirements. This completes the proof of Theorem 2.

\section{Concluding remarks}

The assumption in Theorem 1 that the curves are two-way infinite is not important. If we have a family $\mathcal{F}=\mathcal{A} \cup \mathcal{B}$ of arbitrary $x$-monotone curves such that, for any pair of curves $\alpha \in \mathcal{A}, \beta \in \mathcal{B}$ which can be met by a vertical line, 
$\alpha$ lies above $\beta$, we can make each curve two-way infinite without destroying this property, by adding only at most

$$
2\left(\begin{array}{c}
|\mathcal{A}| \\
2
\end{array}\right)+2\left(\begin{array}{c}
|\mathcal{B}| \\
2
\end{array}\right)<|\mathcal{F}|^{2}
$$

crossings.

One can give an alternative proof of Theorem 1 by reducing it to a combinatorial statement about sequences. Let $\left(x_{1}, \ldots, x_{m}\right)$ be a sequence of $m$ elements taken from a finite alphabet $\Phi$. For any pair of distinct elements $a, b \in \Phi$, define the number of alternations of $a$ and $b$ in the sequence, as the largest number $t$ such that there is a subsequence $\left(x_{i(0)}, x_{i(1)}, \ldots, x_{i(t)}\right)$ of length $t+1$ with $1 \leq i(0)<i(1)<\ldots<i(t) \leq m$ such that its elements alternate between $a$ and $b$ (or between $b$ and $a$ ). That is,

$$
x_{i(0)}=x_{i(2)}=\ldots=a, \quad x_{i(1)}=x_{i(3)}=\ldots=b,
$$

or

$$
x_{i(0)}=x_{i(2)}=\ldots=b, \quad x_{i(1)}=x_{i(3)}=\ldots=a .
$$

This number $t$ is denoted by $\operatorname{alt}_{\{a, b\}}\left(x_{1}, \ldots, x_{n}\right)$.

Define the alternation number of the sequence $\left(x_{1}, \ldots, x_{m}\right)$, as

$$
\sum_{\{a, b\} \subseteq \Sigma} \operatorname{alt}_{\{a, b\}}\left(x_{1}, \ldots, x_{m}\right),
$$

where the sum is taken over all unordered pairs $\{a, b\}$ of distinct elements from $\Phi$.

Theorem 1 can also be proved using the following result, which is perhaps of independent interest.

Theorem 3. Let $\left(x_{1}, \ldots, x_{m}\right)$ be a sequence of length $m$ over an alphabet $\Phi$. Assume that there exists an absolute constant $c>0$ such that for all $1 \leq z \leq$ $m$, every $z$ consecutive elements of the sequence contain at least $c \sqrt{z}$ distinct symbols. Then the alternation number of the sequence $\left(x_{1}, \ldots, x_{m}\right)$ is at least $d m \log m$, for a suitable constant $c^{\prime}>0$, depending only on $c$.

Salazar [8] verified the Richter-Thomassen conjecture in the special case when any pair of curves have at most $k$ points in common, for a fixed constant $k$. The best known general bound is due to Mubayi [3], who proved that any family of $n$ closed curves in general position in the plane determines at least $\left(\frac{4}{5}+o(1)\right) n^{2}$ intersection points.

\section{References}

1. T. M. Chan. On levels in arrangements of curves, II: A simple inequality and its consequences. Discrete \& Computational Geometry, 34(1):11-24, 2005. 
2. P. Koebe. Kontaktprobleme der konforman abbildung. Berichte über die Verhandlungen d. Sächs. Akademie der Wissenschaften Leipzig, 88:141-164, 1936.

3. D. Mubayi. Intersecting curves in the plane. Graphs and Combinatorics, 18(3):583589, 2002.

4. P. Erdős. On sets of distances of $n$ points. The American Mathematical Monthly, 53:248-250, 1946.

5. J. Pach and P. K. Agarwal. Combinatorial Geometry. Wiley, New York, 1995.

6. R. B. Richter and C. Thomassen. Intersection of curves systems and the crossing number of $C_{5} \times C_{5}$. Discrete \& Computational Geometry, 13:149-159, 1995.

7. F. Ruskey and M. Weston. Venn diagram survey. Electronic Journal of Combinatorics, DS\#5, 2005.

8. G. Salazar. On the intersections of systems of curves. Journal of Combinatorial Theory Series B, 75:56-60, 1999.

9. J. Venn. On the diagrammatic and mechanical representation of propositions and reasonings. Philosophical Magazine and Journal of Science, Series 5, 10(59), 1880. 